Send your letters to the Editor,

British Dental Journal,

64 Wimpole Street

London

W1G 8YS

Email bdj@bda.org

Priority will be given to letters less than 500 words long.

Authors must sign the letter, which

may be edited for reasons of space.

\section{AJAX NUMBER}

Sir, I have been surprised when examining dental nurses for the post-qualification sedation exam to note that most candidates have an extremely good knowledge of measuring blood pressure (BP) but rarely note the heart rate (HR) when doing so. There is little doubt as to the potential usefulness of BP when assessing a patient's fitness for dental treatment.

Many authorities recommend the routine monitoring of BP (often every five minutes during dental anaesthesia and sedation). In my 20 years of dental general anaesthesia (DGA) practice and 30 years of dental sedation (DS) practice I have routinely measured BP and HR throughout in addition to ECG, oxygen saturation and expired carbon dioxide etc. While a snap shot BP can give warning to a clinician with regard to possible heart failure etc, it needs to be related to HR to estimate trends in cardiac output (CO) and probably more usefully stroke volume (SV).

To this end considering dental anxiety and the influence of adrenalin on the heart, many sedationists would consider this a useful thing to know.

For the past ten years, we have been routinely measuring the difference between Systolic pressure (in $\mathrm{mm} \mathrm{Hg}$ ) and HR and have found that a satisfactorily sedated patient will have a value of very approximately 30 or less (frequently at the start of a DS with a very anxious patient we will record systolic pressures of approx 170 and a HR of approximately 90 . The value we note at the start of the procedure would therefore be 80 . Once adequately sedated HR would drop to say 80 and systolic pressure to 110 giving a new value of 30 ). We have been calling this value the 'AJAX' number. Interestingly, a retrospective study of DGA patients shows a similar value in our practice.

We have found this to be a very useful value to titrate increments of eg Propfol during long sedations. Obviously there are many influencing factors not to mention medication specifically to control BP.

I would be interested to hear if anyone else has used this method as I have seen little reference in the literature.

Incidentally the choice of name is not that I am a classicist but rather it's a corruption of my name (Arthur Jack). Sad eh?

A. Jack

DOI: 10.1038/sj.bdj2010.631

\section{HERBAL INTERACTIONS}

Sir, we wish to highlight an unusual case of post-operative bleeding following dental extraction that presented to the Accident and Emergency department at Manchester Royal Infirmary.

A 46-year-old Caucasian male presented complaining of continued bleeding from the operative site where he had undergone extraction of the upper right first permanent molar earlier that day. The patient stated the extraction was uneventful and the tooth was removed as a whole by his GDP. Applying pressure to the area had not stopped the bleeding.

The patient's medical history was unremarkable. He was taking paracetamol $1 \mathrm{~g}$ and ibuprofen $400 \mathrm{mg}$ for pain relief as prescribed by his GDP. However, on further questioning the patient revealed that, for a number of years, he had been taking a host of vitamins and herbal remedies including Ginkgo Biloba, ginseng, garlic and ginger.
On examination poor clot formation was noted about the surgical site with a gentle ooze of blood present.

The bleeding was arrested with local measures including an oxidised cellulose dressing (Surgicel) and resorbable sutures over the socket (Vicryl 3.0).

Whilst there are a number of case studies reporting post-operative bleeding in patients taking herbal remedies such as Ginkgo Biloba, few relate to dental surgery. ${ }^{1-5}$

This case further highlights the potential for post-operative complications and drug interactions in patients taking herbal remedies. A thorough medical and drug history must be taken from all patients. Unfortunately many patients feel they do not need to inform health professionals that they take herbal remedies, as they do not perceive them to be medically relevant.

N. Patel

By email

1. Bent S, Goldberg H, Padula A, Avins A Spontaneous bleeding associated with ginkgo biloba: a case report and systematic review of the literature. J Gen Intern Med 2005; 20: 657-661.

2. Destro M W, Speranzini M B, Cavalheiro Filho $C$ Destro T, Destro C. Bilateral haematoma after rhytidoplasty and blepharoplasty following chronic use of Ginkgo biloba. Br J Plast Surg 2005: 58: 100-101.

3. Meisel C, Johne A, Roots I. Fatal intracerebral mass bleeding associated with Ginkgo biloba and ibuprofen. Atherosclerosis 2003; 167: 367.

4. Little J W. Complementary and alternative medicine: impact on dentistry. Oral Surg Oral Med Oral Pathol Oral Radiol Endod 2004; 98: 137-145

5. Fessenden J M, Wittenborn W, Clarke L. Ginkgo biloba: a case report of herbal medicine and bleeding postoperatively from a laparoscopic cholecystectomy. Am Surg 2001; 67: 335

DOI: 10.1038/sj.bdj.2010.632

\section{PURPLE HAZE}

Sir, I am writing this letter in regard to a patient in whom I placed a left sided inferior dental nerve block in order to 
carry out a crown preparation of an LL7. The first block did not take fully so therefore I placed a second block which was placed higher.

As half the anaesthetic went in the patient complained of double vision and flashing lights which turned from being dark purple to a light purpley haze.

I immediately stopped the injection and tried to reassure the patient. On examining her right eye, the eye was looking mesially, possibly compensating for blocking one of the nerves to the left eye muscles.

I was wondering if anybody else has had this phenomenon with colour and flashing lights. I was under the impression that the optic nerve was involved in vision and I was not aware from doing anatomy many years ago that a dental anaesthetic could block this nerve! In fact I feel there must be another reason why this occurrence happened. We carried on with the crown prep after reassuring the patient and after 15 minutes things settled back to normal.

\section{Hughes \\ Anglesey \\ DOI: 10.1038/sj.bdj.2010.633}

\section{TOOLKIT PROCEDURES}

Sir, I would like to comment on the recent paper Caries control in health service practice by Page et al. (BDJ 2010; 208: 449-450), concerning the guidelines in the DH Oral Health toolkit.

My NHS practice is predominantly concerned with the treatment of children. I have been following the toolkit guidelines, including the application of fluoride varnish twice a year to most 3-18-year-old patients, including those at low risk of caries and more frequently to those patients at high risk. At the same time I have been attempting to follow the NICE guidelines on oral health assessments for low risk young patients, increasing the time intervals to nine months or a year, as recommended.

1. I have found that parents seem to appreciate the additional preventative measures provided for their children

2. The authors of the paper are correct, it takes longer to carry out the examination, oral hygiene instruction, cleaning and the varnish application for these patients. The treatment schedule is slowed, so that for the first time since 2006 I have missed my UDA target by about 10\%. Unless I have a sympathetic PCT, it is likely that my NHS income will be similarly reduced. In addition, additional costs have been incurred for materials

3. Fewer band 2 treatments are being carried out, either because the varnish is preventing decay, or because of the slow down in treatment times. Again, it becomes harder to reach the UDA target

4. For low risk patients, the NICE recall and the DH toolkit guidelines cannot be reconciled. For example, say a nine month recall is appropriate, then:

Month 1 Assessment and varnish

Month 6 Varnish

Month 9 Assessment

Month 12 Varnish

Month 18 Assessment and varnish

Month 24 Varnish

Month 27 Assessment

Month 30 Varnish.

The NICE recall assessment guidelines were issued in 2004, long before the oral health toolkit recommendations. NICE therefore needs to review its guidance, to ensure there is no conflict

5. Further to the above, the NHS claim form has recently been amended to include a box which has to be completed stating that the dentist has complied with the NICE guidelines and provided all necessary preventative treatment. If the guidelines cannot always be reconciled, how can the box be completed?

6. In view of the doubts expressed in the recent paper concerning the cost effectiveness of applying fluoride varnish to low risk patients, perhaps NICE should review this new procedure, before it is widely implemented.

S. S. Lawson Pinner DOI: 10.1038/sj.bdj.2010.634

\section{BRIDGING THE GAP}

Sir, We would like to share our experience as an oral and maxillofacial surgery
(OMFS) Senior House Officer (SHO) and a Specialty Trainee Doctor in Paediatrics.

As stated by Harris et al., ${ }^{1}$ dental professionals as well as those committed to paediatric dentistry find child protection to be a difficult and challenging area of work with a need for improved child protection training for dental professionals.

OMFS SHOs around the UK are regularly exposed to a considerable number of cases of facial and dental trauma in paediatric patients during on-call duties, often covering several hospital sites. Emergency departments can experience extremely busy times and although in most cases children are initially assessed by experienced nurses and doctors, on occasion OMFS SHOs are the first health contact. For example, expected admissions from peripheral hospitals and outof-hours dental referrals bypass initial triage. In these cases the responsibility for assessing child protection lies with the on-call OMFS SHO. But are we well prepared for that?

Within these circumstances, we write to remark how crucial child protection awareness is, the need of establishing good rapport with the child in order to extract first hand information and a comprehensive history taking including a detailed family and social history. A systematic facial and dental examination and high standard of record keeping are also valuable in identifying or alleviating concerns.

Our observation is that oro-dental examination is often poorly documented and not comprehensively referred by emergency department staff. As a paediatric doctor working in a children's emergency department, I have seen first hand that basic dental knowledge is lacking from both adult and paediatric doctors.

In these clinical settings, how many times are children seen by professionals who are not appropriately trained to assess child protection? How often do people ask for help in this matter or know the appropriate referral pathway? Who should have the responsibility in ensuring a child is in a place of safety and has not been subject to abuse?

Although child protection training in secondary care is regularly offered to all staff in contact with children, the regulation of this is well overseen in paediat- 